\title{
Craniotomia descompressiva não osteoplástica na lesão difusa do tipo IV de Marshall - Relato de caso e discussão
}

\section{Decompression Craniotomy not Osteoplastic in Diffuse Injury Type IV Marshall - Case Report and Discussion}

\author{
Jefferson Rosi Junior ${ }^{1}$ Eberval Gadelha de Figueiredo \\ Lin Tchia Yeng ${ }^{5}$ Manoel Jacobsen Teixeira ${ }^{6}$ \\ ${ }^{1}$ Neurocirurgião do Hospital das Clínicas da Faculdade de Medicina da \\ Universidade de São Paulo (HC-FM-USP) e do Conjunto Hospitalar do \\ Mandaqui (CHM), São Paulo, SP, Brasil \\ 2 Professor da Divisão de Neurocirurgia do HC-FM-USP, São Paulo, SP, Brasil \\ ${ }^{3}$ Médico residente em Neurocirurgia do CHM, São Paulo, SP, Brasil \\ ${ }^{4}$ Médica residente em Medicina Física e Reabilitação da Escola Paulista de \\ Medicina, Universidade Federal de São Paulo (Unifesp), São Paulo, SP, Brasil \\ ${ }^{5}$ Chefe do ambulatório de Dor em Fisiatria do HC-FM-USP, São Paulo, \\ SP, Brasil \\ 6 Professor Titular da Divisão de Neurocirurgia do HC-FM-USP, São \\ Paulo, SP, Brasil
}

Arq Bras Neurocir 2015;34:68-72.

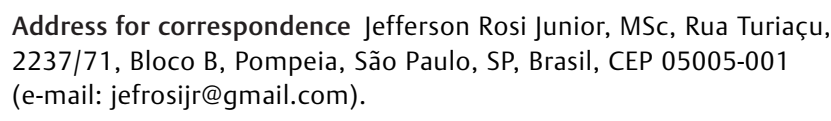

Address for correspondence Jefferson Rosi Junior, MSc, Rua Turiaçu, 2237/71, Bloco B, Pompeia, São Paulo, SP, Brasil, CEP 05005-001 (e-mail: jefrosijr@gmail.com).

Neurocirurgia do Hospital das Clínicas da Faculdade de Medicina da Universidade de São Paulo, São Paulo, SP, Brasil e do Conjunto Hospitalar do Mandaqui, São Paulo, SP, Brasil

\author{
Resumo \\ Palavras-Chave \\ - traumatismos \\ encefálicos \\ - tomografia \\ computadorizada por \\ raios $\mathrm{X}$ \\ - neurocirurgia \\ - hipertensão \\ intracraniana \\ - craniectomia \\ descompressiva
}

Os autores apresentam um caso de tumefação encefálica difusa pós-traumática, em que a paciente em coma, com 6 pontos na escala de Glasgow e anisocórica, foi submetida à craniotomia descompressiva não osteoplástica, com resultado satisfatório e evolução para Glasgow de 15 pontos no sétimo pós-operatório. received

October 31, 2014

accepted

November 1, 2014
DOI http://dx.doi.org/

$10.1055 / \mathrm{s}-0035-1547390$ ISSN 0103-5355.
Copyright @ 2015 Thieme Publicações

Ltda, Rio de Janeiro, Brazil
License terms

(c) $(1) \$$ 


\author{
Abstract \\ Keywords \\ - brain injuries \\ - tomography X-ray \\ computed \\ - neurosurgery \\ - intracranial \\ hypertension \\ - decompressive \\ craniectomy
}

The authors show a patient with post traumatic encephalic swelling, with 6 points at Glasgow scale on admission. Decompressive craniectomy was performed and the patient has a good outcome, with 15 points at the Glasgow scale, seven days after the neurosurgery.

\section{Introdução}

O traumatismo cranioencefálico (TCE) continua a proporcionar situações críticas de saúde, com numerosos casos todos os anos e importantes morbidade e mortalidade. Afeta todas as faixas etárias, com maior incidência no sexo masculino, sobretudo nas faixas etárias economicamente ativas, ao passo que nos extremos de vida, tende a acometer homens e mulheres de modo mais uniforme. ${ }^{1-6}$

As quedas de altura, as ocorrências relacionadas ao trânsito e as agressões são as causas mais comuns doTCE. Decorrentes do TCE, lesões com potencial necessidade de abordagem neurocirúrgica podem advir, acompanhadas de quadros de deterioração das funções neurológicas e até de coma, e exigem não só o tratamento operatório, como também os cuidados intensivos neurológicos no pós-operatório. ${ }^{1-6}$

Nem sempre a neurocirurgia é praticada no intuito de se retirar um volumoso hematoma intracraniano que proporciona aumento da pressão intracraniana (PIC). Quadros de tumefação encefálica puros, como os descritos na classificação de Marshall ( - Tabela 1), também podem necessitar de uma intervenção neurocirúrgica, foco do presente artigo.

\section{Relato de Caso}

Paciente do sexo feminino, 31 anos, caiu da escada por volta das 16 horas do dia 24 de outubro de 2011, e pelo resgate foi encontrada no local de queda com Glasgow de 3 pontos, e então conduzida já sob ventilação mecânica ao prontosocorro (PS) do Conjunto Hospitalar do Mandaqui (CHM).
No PS foi admitida com Glasgow de 6 pontos, com pupilas anisocóricas, sendo a pupila esquerda maior que a direita, com reflexo fotomotor direto e consensual presente, porém retardado à esquerda, sem relato de uso de sedativos para realizar-se a entubação. Três familiares foram questionados sobre a existência de anisocoria fisiológica prévia, e todos a negaram.

Realizada tomografia computadorizada do craniencéfalo (TCC), verificou-se tumefação encefálica difusa, sobretudo no hemisfério cerebral esquerdo, com desvio das estruturas de linha mediana de $0,5 \mathrm{~cm}$. 0 sistema ventricular apresentava dimensões reduzidas, e não se visualizava as cisternas basais e os sulcos corticais.

Diante do quadro neurológico e radiológico, foi indicada a realização de craniotomia descompressiva não osteoplástica (CDNO), também denominada craniectomia descompressiva, iniciando-se o procedimento 4 horas após o TCE.

A neurocirurgia foi realizada por meio de incisão cutânea frontotemporoparietal e craniotomia de seis furos, pelo uso de trépano manual e serra de Gigli, não sendo aberta a duramáter. Um dreno a vácuo foi deixado no espaço epidural por prevenção de hematoma epidural pós-operatório, pois a paciente ficou sem o flap ósseo. As - Figs. 1, 2 e 3 referemse à TCC pré-operatória e as -Figs. $\mathbf{4 , 5}$ e $\mathbf{6}$ ao terceiro pósoperatório, não tendo sido feita TCC antes do terceiro dia após a cirurgia.

Até o terceiro pós-operatório a paciente foi mantida sedada com midazolam e fentanil, e no sétimo pós-operatório, encontrou-se a paciente ainda na unidade de terapia intensiva (UTI) com Glasgow de 15, sem déficits neurológicos

Tabela 1 Classificação de Marshall por tomografia do traumatismo craniencefálico

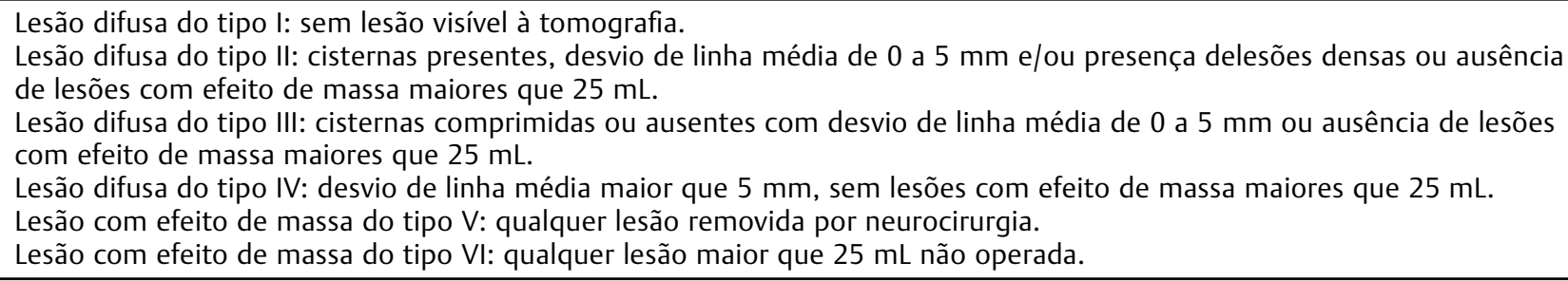




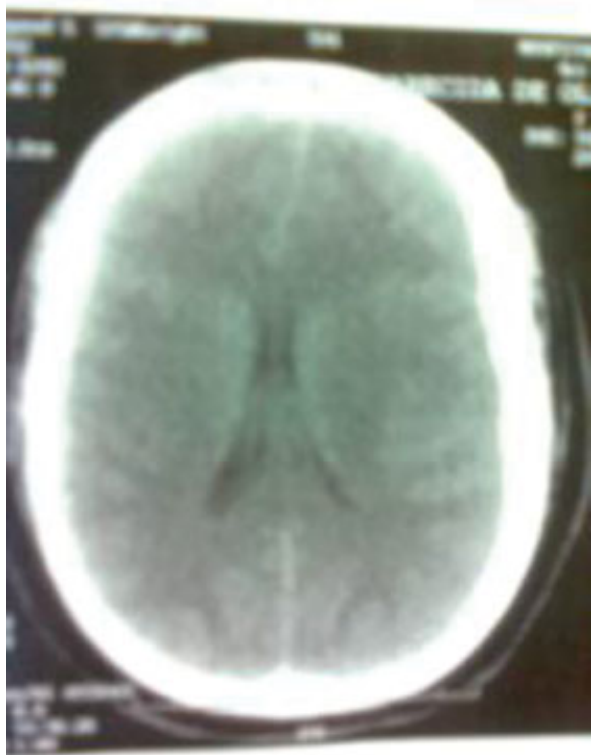

Fig. 1 Corte axial de tomografia do encéfalo à altura do sistema ventricular demonstra desvio discreto das estruturas da linha mediana, tumefação encefálica e compressão do sistema ventricular, encontrados na lesão difusa do tipo IV de Marshall.

grosseiros, e em 7 de novembro de 2011 a paciente recebeu alta hospitalar.

\section{Discussão}

Trata-se de um caso isolado, e uma série significativa de casos é necessária para maiores conclusões.

Os neurocirurgiões devem levar em consideração que não somente os volumosos hematomas intracranianos necessitam de operação. Casos de tumefação encefálica - sobretudo os casos de lesão difusa do tipo IV de Marshall, quando

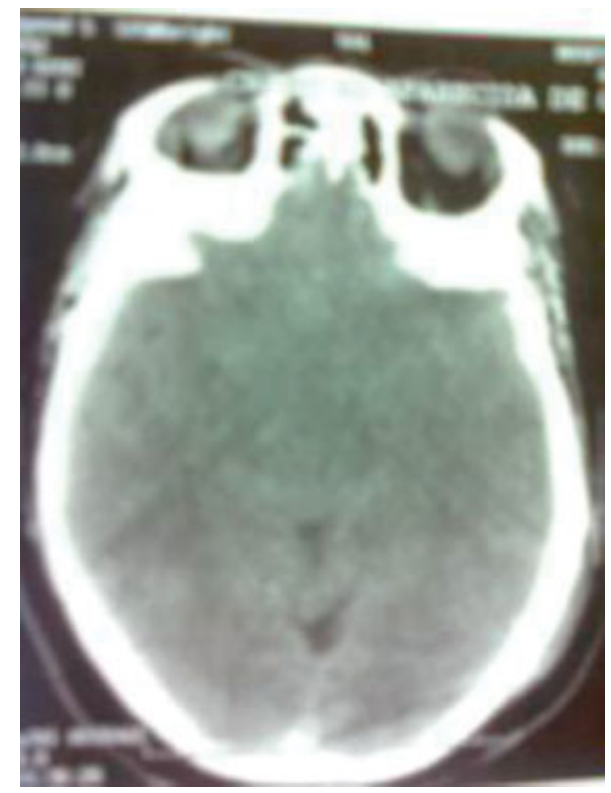

Fig. 2 Corte axial de tomografia do crânio ao nível das cisternas basais mostra apagamento cisternal basal, típico da lesão difusa do tipo IV de Marshall.

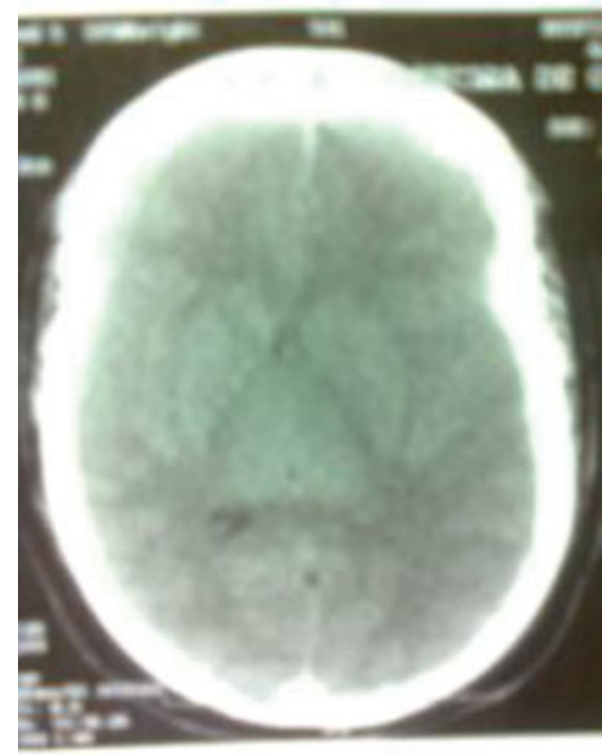

Fig. 3 Corte axial de tomografia do encéfalo à altura do sistema ventricular demonstra desvio discreto das estruturas da linha mediana, tumefação encefálica e importante compressão do sistema ventricular, encontrados na lesão difusa do tipo IV de Marshall.

predomina o inchaço cerebral em um dos hemisférios, com desvio de estruturas de linha mediana e potencial para herniação - podem também necessitar de uma intervenção neurocirúrgica com intuito de baixar a pressão intracraniana e salvaguardar a vida do paciente, sendo que quanto mais precoce for sua realização melhor tende a ser o prognóstico de sobrevida do paciente, bem como sua recuperação funcional. No caso abordado, a operação foi realizada 4 horas

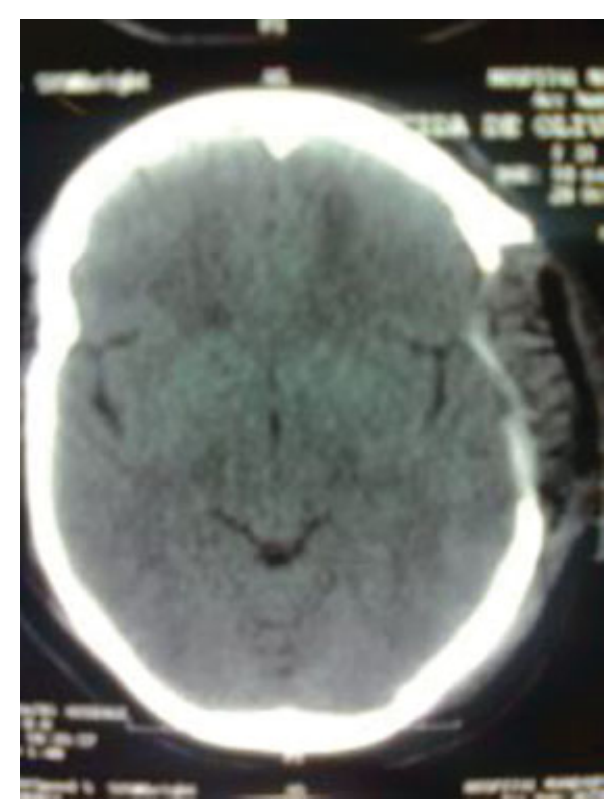

Fig. 4 Corte axial de tomografia do crânio no primeiro pós-operatório, com a craniotomia não osteoplástica realizada, onde já se observa aparecimento da cisterna sylviana, inferindo indiretamente queda da pressão intracraniana. 


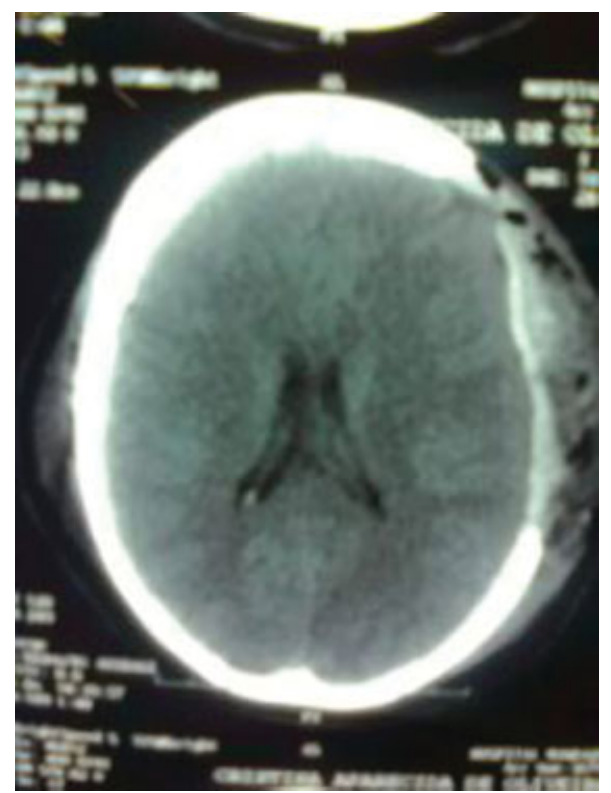

Fig. 5 Corte axial de tomografia do encéfalo à altura do sistema ventricular demonstra, no primeiro pós-operatório, a craniotomia realizada e o maior volume do sistema ventricular menos comprimido em relação ao pré-operatório.

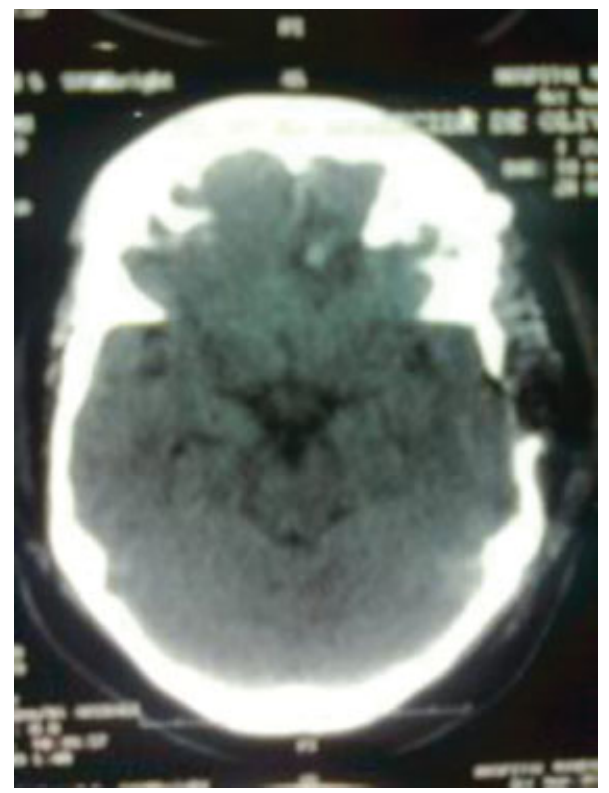

Fig. 6 Corte axial de tomografia do encéfalo à altura das cisternas basais que no primeiro pós-operatório passaram a ser visíveis.

após o TCE, e isto certamente colaborou para o bom resultado.

Casos de pacientes operados muito tardiamente não tendem a ter um prognóstico tão favorável quanto o conseguido neste caso, visto que o longo período de hipertensão intracraniana com má perfusão sanguínea encefálica é campo fértil para o surgimento da isquemia e infartos encefálicos secundários. ${ }^{6-8}$
Julgamos que não seria fácil um comitê de ética em pesquisa médica autorizar a realização de um estudo comparativo de morbidade e mortalidade em um grupo de tratamento exclusivo de UTI neurológica, com aferições da pressão intracraniana (PIC) versus outro grupo primeiramente submetido a um procedimento neurocirúrgico e depois tratado na UTI.

O que teria maior facilidade em ser liberado seria uma comparação entre duas diferentes neurocirurgias: craniotomia em um grupo de pacientes versus derivação ventricular externa com drenagem contínua do líquor e aferição da PIC em outro grupo; ou ainda outro grupo que passe por ambas as intervenções, seguidas dos cuidados de UTI neurológica em quaisquer dos grupos.

O controle da PIC na UTI é facilitado quando se tem um paciente previamente submetido à craniotomia descompressiva, e em muitas situações a incapacidade de controlar a pressão intracraniana com medidas clínicas indica a realização da craniotomia descompressiva como medida heroica. ${ }^{7-12}$

A TCC no pós-operatório costuma mostrar abertura das cisternas basais, visualização mais fácil dos sulcos corticais e retorno a padrões mais próximos do fisiológico da morfologia do sistema ventricular, que são achados compatíveis com o retorno da PIC aos padrões aceitáveis. O que se tinha no exame de TCC pré-operatório da paciente era justamente o contrário. $^{2,4-6,13,14}$

Quando se tem disponível o doppler transcraniano (DTC) na emergência, como no caso descrito, ele costuma mostrar no primeiro dia padrão hemodinâmico encefálico de oliguemia, ou seja, de diminuição das velocidades de fluxo sanguíneo nas grandes artérias encefálicas de modo difuso, e por vezes até velocidades de fluxo sanguíneo ainda menores no hemisfério cerebral de maior tumefação, com índices de pulsatilidade elevados, refletindo o aumento da resistência vascular encefálica, e sendo compatível com os achados de imagem da TCC, muito sugestivos de aumento considerável da PIC.

No pós-operatório de craniotomia descompressiva, o DTC tende a mostrar queda dos índices de pulsatilidade e aumento das velocidades de fluxo sanguíneo encefálico, saindo assim de uma situação de oliguemia pré-operatória, ou até de certa hiperemia que também pode ser verificada no primeiro dia de TCE, para um padrão de hiperemia intensa que pode perdurar por semanas, com ou sem período de vaso espasmo subsequente, para no final dar lugar a normalização dos padrões de índices de pulsatilidade e de velocidades de fluxo sanguíneo. $6,15,16$

A realização da craniotomia descompressiva sem a abertura de dura-máter, como praticado no caso em questão, tem por objetivo evitar a extrusão do tecido encefálico tumefeito pela abertura da craniotomia, e tenta também minimizar o risco de meningites e de ventriculites, devido a abertura dural, e sobretudo quando é inserido um cateter ventricular para drenagem de líquor e o mesmo necessite permanecer no paciente por períodos prolongados (mais de 3 dias), em que as chances de um paciente evoluir com ventriculite são menores, comparadas às dos pacientes que necessitem fazer 
uso de cateteres de derivação ventricular externa por mais de 5 dias. ${ }^{6,7,17}$

A abertura dural certamente pode diminuir ainda mais a PIC em relação a uma neurocirurgia sem a abertura da dura-máter, porém requer um tempo neurocirúrgico mais prolongado no fechamento pela necessidade de realizar plástica da dura máter, no caso da sutura da gálea aponevrótica, ou de fáscia junto a dura-máter, e custo mais elevado, no caso de se optar pelo uso da plástica com as duras-máteres artificiais. ${ }^{6,8-11,18}$

A craniotomia descompressiva no molde realizado no caso descrito é um procedimento de rápida execução, não superior a 40 minutos, e deve ser ampla o suficiente para proporcionar alívio da hipertensão intracraniana, focando principalmente a liberação das porções mais inferiores, anteriores e mediais do lobo temporal - que podem dar origem a hérnias cerebrais com potencial para comprimir o tronco encefálico e levar o paciente a óbito -, o que é conseguido com o feitio da craniotomia o mais rasante possível no osso temporal. ${ }^{6-13,18}$

Passada a fase de tumefação, cranioplastia deve ser realizada para proteção do tecido encefálico e melhora do padrão estético, o que pode ser realizado por meio do reimplante do próprio osso do paciente, armazenado em banco de ossos, ou do tecido celular subcutâneo da parede abdominal, ou mesmo de próteses de metilmetacrilato, de titânio, ou das mesclas de titânio com hidroxiapatita.9,19-25

A craniotomia descompressiva não osteoplástica, também dita craniectomia, é uma possibilidade terapêutica para os casos de lesão difusa do tipo IV de Marshall. Há necessidade de maiores casuísticas e de comparações com outras modalidades de tratamento para que se confirmem as suspeitas de que a craniotomia descompressiva não osteoplástica, além de ser um procedimento de rápida execução, seja um procedimento mais seguro do ponto de vista infeccioso e capaz de evitar a hernição do encéfalo para além das bordas da craniotomia.

\section{Conflitos de Interesse}

Os autores declaram não haver conflitos de interesse.

\section{Referências}

1 Cameron PA, Rainer TH, Mak P. Motor vehicle deaths in Hong Kong: opportunities for improvement. J Trauma 2004;56(4): 890-893

2 Hukkelhoven CW, Steyerberg EW, Rampen AJ, et al. Patient age and outcome following severe traumatic brain injury: an analysis of 5600 patients. J Neurosurg 2003;99(4):666-673

3 Mar J, Arrospide A, Begiristain JM, Larrañaga I, Elosegui E, OlivaMoreno J. The impact of acquired brain damage in terms of epidemiology, economics and loss in quality of life. BMC Neurol 2011;11:46

4 Martins ET, Linhares MN, Sousa DS, et al. Mortality in severe traumatic brain injury: a multivariated analysis of 748 Brazilian patients from Florianópolis City. J Trauma 2009;67(1):85-90

5 McArthur DL, Chute DJ, Villablanca JP. Moderate and severe traumatic brain injury: epidemiologic, imaging and neuropathologic perspectives. Brain Pathol 2004;14(2):185-194
6 Rosi J Júnior. Neurônio: perguntas e respostas em neurocirurgia. São Paulo: Scortecci; 2011

7 Guerra WK, Gaab MR, Dietz H, Mueller JU, Piek J, Fritsch MJ. Surgical decompression for traumatic brain swelling: indications and results. J Neurosurg 1999;90(2):187-196

8 Polin RS, Shaffrey ME, Bogaev CA, et al. Decompressive bifrontal craniectomy in the treatment of severe refractory posttraumatic cerebral edema. Neurosurgery 1997;41(1):84-92

9 Josan VA, Sgouros S. Early decompressive craniectomy may be effective in the treatment of refractory intracranial hypertension after traumatic brain injury. Childs Nerv Syst 2006;22(10): 1268-1274

10 Quinn TM, Taylor JJ, Magarik JA, Vought E, Kindy MS, Ellegala DB. Decompressive craniectomy: technical note. Acta Neurol Scand 2011;123(4):239-244

11 Rosenfeld JV, Cooper J. What is the role for decompressive craniectomy in severe traumatic brain injury? Re: Decompressive craniectomy: surgical control of intracranial hypertension may improve outcome. Injury 2010;41(9):899-900

12 Rosi J Júnior, Oliveira PGD, Montanaro AC, Godoy R. Craniotomia descompressiva em doença cérebro-vascular isquêmica extensa supratentorial. An Paul Med Cir 2002;129:76-81

$13 \mathrm{Kim} \mathrm{JJ}$, Gean AD. Imaging for the diagnosis and management of traumatic brain injury. Neurotherapeutics 2011;8(1):39-53

14 Maas AI, Hukkelhoven CW, Marshall LF, Steyerberg EW. Prediction of outcome in traumatic brain injury with computed tomographic characteristics: a comparison between the computed tomographic classification and combinations of computed tomographic predictors. Neurosurgery 2005;57(6): 1173-1182

15 Bor-Seng-Shu E, Aguiar PH, de Almeida Leme RJ, Mandel M, Andrade AF, Marino R Jr. Epidural hematomas of the posterior cranial fossa. Neurosurg Focus 2004;16(2):ECP1

16 Bor-Seng-Shu E, Hirsch R, Teixeira MJ, De Andrade AF, Marino R Jr. Cerebral hemodynamic changes gauged by transcranial Doppler ultrasonography in patients with posttraumatic brain swelling treated by surgical decompression. J Neurosurg 2006;104(1): 93-100

17 Honeybul S. Complications of decompressive craniectomy for head injury. J Clin Neurosci 2010;17(4):430-435

18 Huang X, Wen L. Technical considerations in decompressive craniectomy in the treatment of traumatic brain injury. Int J Med Sci 2010;7(6):385-390

19 Asano Y, Ryuke Y, Hasuo M, Simosawa S. Cranioplasty using cryopreserved autogenous bone. No To Shinkei 1993;45(12): $1145-1150$

20 Belmahi A, Gharib NE, Bencheikh R, Abbassi A, Mizahi M. Reconstruction of large scalp and calvarium defects by using the semifree latissimus dorsi flap associated with methylmethacrylate implant for cranioplasty. Ann Chir Plast Esthet 2002;47(4): 298-303

21 Dujovny M, Aviles A, Agner C, Fernandez P, Charbel FT. Cranioplasty: cosmetic or therapeutic? Surg Neurol 1997;47(3): 238-241

22 Ducic Y. Titanium mesh and hydroxyapatite cement cranioplasty: a report of 20 cases. J Oral Maxillofac Surg 2002;60(3):272-276

23 Joffe J, Harris M, Kahugu F, Nicoll S, Linney A, Richards R. A prospective study of computer-aided design and manufacture of titanium plate for cranioplasty and its clinical outcome. $\mathrm{Br} \mathrm{J}$ Neurosurg 1999;13(6):576-580

24 Luparello D, Bruschi S, Verna G, et al. Cranioplasty with polymethylmethacrylate. The clinico-statistical considerations. Minerva Chir 1998;53(6):575-579

25 Rosi J Júnior, Oliveira PGD, Montanaro AC, Godoy R. Cranioplastia autóloga pós craniectomia descopressiva: Análise prospectiva. An Paul Med Cir 2005;132:19-22 